Hard X-ray Imaging for Measuring Laser Absorption Spatial Profiles on the National Ignition Facility

E. L. Dewald, O. S. Jones, O. L. Landen, L. Suter, P. Amendt, R. E. Turner, S. Regan

May 5, 2006

High Temperature Plasma Diagnostics Williamsburg, VA, United States May 7, 2006 through May 11, 2006 
This document was prepared as an account of work sponsored by an agency of the United States Government. Neither the United States Government nor the University of California nor any of their employees, makes any warranty, express or implied, or assumes any legal liability or responsibility for the accuracy, completeness, or usefulness of any information, apparatus, product, or process disclosed, or represents that its use would not infringe privately owned rights. Reference herein to any specific commercial product, process, or service by trade name, trademark, manufacturer, or otherwise, does not necessarily constitute or imply its endorsement, recommendation, or favoring by the United States Government or the University of California. The views and opinions of authors expressed herein do not necessarily state or reflect those of the United States Government or the University of California, and shall not be used for advertising or product endorsement purposes. 


\title{
Hard $x$-ray imaging for measuring laser absorption spatial profiles on the National Ignition Facility*
}

\author{
E.L. Dewald, O.S. Jones, O.L. Landen, L. Suter, P.Amendt, R.E. Turner \\ Lawrence Livermore National Laboratory, P.O. Box 808, Livermore, CA 94551, USA \\ S. Regan
}

U. Rochester, LLE, USA

\section{Abstract}

Hard x-ray ("Thin wall") imaging will be employed on the National Ignition Facility (NIF) to spatially locate laser beam energy deposition regions on the hohlraum walls in indirect drive Inertial Confinement Fusion (ICF) experiments, relevant for ICF symmetry tuning. Based on time resolved imaging of the hard x-ray emission of the laser spots, this method will be used to infer hohlraum wall motion due to $\mathrm{x}$-ray and laser ablation and any beam refraction caused by plasma density gradients. In optimizing this measurement, issues that have to be addressed are hard x-ray visibility during the entire ignition laser pulse with intensities ranging from $10^{13}$ to $10^{15} \mathrm{~W} / \mathrm{cm}^{2}$, as well as simultaneous visibility of the inner and the outer laser drive cones. In this work we will compare the hard x-ray emission calculated by LASNEX and analytical modeling with thin wall imaging data recorded previously on Omega and during the first hohlraum experiments on NIF. Based on these calculations and comparisons the thin wall imaging will be optimized for ICF/NIF experiments. 


\section{Introduction}

Hard x-ray imaging (also known as "thinwall imaging") is an experimental technique used to measure hot plasma emission inside hohlraums ${ }^{1}$. The chosen high- $Z$ hohlraum wall thickness, in the 2-10 um range contains the thermal x-rays used to drive a fuel capsule in indirect drive inertial confinement fusion (ICF) experiments ${ }^{2}$, but is transparent to the hard $\mathrm{x}$-ray $(>9 \mathrm{keV})$ emission of the laser spots. Since the ICF hohlraums contain a low-Z fill to slow down the inward motion of the heated high- $Z$ plasma, the hard x-ray emission is localized near the high $Z$ walls. This space and timeresolved emission can then be used to infer motion of the laser plasma re-emission spot on the walls due to a combination of inward heated wall motion and beam refraction which can affect radiation symmetry at the ICF capsule.

There are several aspects that we have to take into consideration when we study the feasibility of this technique for ICF hohlraums ${ }^{2}$ on the National Ignition Facility ${ }^{3}$ (NIF) where multi-cone laser beam illumination with shaped pulses is used. First, the laser intensity varies over approximately two orders of magnitude during the $15 \mathrm{~ns}$ long ICF pulse. Secondly, the different laser cones in the ICF design have different incidence angles relative to the hohlraum wall surface $(23.5,30,44.5$ and-50 $)$. Consider the $\mathrm{x}$-ray emissivity $\sim \mathrm{n}_{\mathrm{TP}}^{2}$ localized around the turning point density $\mathrm{n}_{\mathrm{TP}}=\mathrm{n}_{\mathrm{c}} \cdot \sin ^{2} \theta$, where the laser suffers total reflection, where $n_{c}\left[\mathrm{~cm}^{-3}\right]=1.110^{21} / \lambda^{2}\left[\mu \mathrm{m}^{2}\right]$ is the critical density for laser light of wavelength $\lambda$ and $\theta$ is the laser beam angle to the hohlraum wall. Assuming either a linear or exponential electron density profile, it is easy to show that the optically thin emissivity at fixed temperature per unit axial length should scale as $\sin ^{6} \theta$ or $\sin ^{4} \theta$ in 
the absence of strong laser absorption before reaching this density ${ }^{4}$. This simple scaling is compared to experiments performed at the Omega laser facilities and detailed $2 \mathrm{~d}$ LASNEX calculations of NIF-ICF experiments.

\section{Multi-cone thin wall imaging experimental data}

Recently $>250 \mathrm{eV}$ vacuum thin wall (5 um) single-ended Au hohlraums were performed using multi-cone smoothed laser beams at the Omega laser facility. In these experiments 20 beams were used, with 1ns flattop laser pulse, energy of $500 \mathrm{~J} /$ beam and 300 by 200 $\mu \mathrm{m}$ spot size disposed in three cones (Fig. 1).

The optically thin hard x-ray emission of laser spots from both far and close hohlraum wall was observed using a gated x-ray camera, 75 um resolution element, filtered for photon energies $>7 \mathrm{keV}$. The x-ray flux of the $42^{\circ}$ beams is about $2.7-6$ times dimmer than that of the $59^{\circ}$ ones, in good agreement with the 2.7-4.4 expected by $\sin ^{4} \theta$ or $\sin ^{6} \theta$ scaling (Fig. 1b).

The wall areas not illuminated by laser beams have an x-ray emission which is about $10 \%$ of that in the spots and is probably due to thermal x-ray heating as estimated in ${ }^{1}$ which hinders the visibility of $21.4^{\circ}$ beams, expected to be $10 \mathrm{x}$ lower. Time integrated $\mathrm{x}-$ ray images of thin wall hohlraums with implosion capsules (Fig. 2a) confirmed the time resolved data.

Thin wall imaging was also employed in the first hohlraum experiments on $\mathrm{NIF}^{6}$ and the data is used for a first experimental assessment of x-ray emission dependence on laser intensity. In these experiments vacuum thin wall (5 um) halfraums and normal incidence flattop laser beams with variable laser power were used. These results (Fig. 2b) show that 
the hard $\mathrm{x}$-ray emission scales linearly with the laser intensity for $\geq 10^{15} \mathrm{~W} / \mathrm{cm}^{2}$ intensities, in agreement with theoretical predictions ${ }^{1}$.

\section{Calculated thin wall emissivities for the NIF-ICF design}

In our first attempt to develop a thin wall diagnostic for NIF, we used 2D LASNEX calculations $^{7}$ of the ICF design using $1 \mathrm{MJ}$ of laser energy and a hohlraum with Laser Entrance Hole (LEH) shields ${ }^{8}$ to calculate the time dependent hard x-ray emission for both the inner and the outer laser beam cones. Figure 3 a shows the total laser power for the inner and the outer cones, and Fig. 3b-the corresponding total and filtered x-ray emission for both inner and outer cones. As expected, the total x-ray emission is proportional to the laser power with a conversion efficiency of about $80 \%$. However, the higher the photon energy threshold due to filtering, the stronger is the x-ray emission variation with the laser intensity. As shown in Fig. 3b, except for the foot of the laser pulse, the x-ray emission ratio between the outer and the inner cones, transmitted through 5 um thick high-Z cocktail $(\mathrm{U}: \mathrm{Au}=0.75: 0.25)$ wall, is in the order of 10-20. Taking into account that the total inner-to-outer cone laser power at the wall is about 2 on the peak of the laser pulse, the scaled x-ray emissivity ratio is about $20-40$ compared to the factor of 14-50 given by $\sin ^{4} \theta$ or $\sin ^{6} \theta$ scaling (Fig. 1b) for the innermost $\left(23.5^{\circ}\right)$ and outermost $\left(50^{\circ}\right)$ laser cones. It has to be noted that the x-ray emission of the inner and outer rings, calculated from the post-processed images shown in Figure 3c, is a result of two sets of laser cones for each ring, i.e. $23.5^{\circ}$ and $30^{\circ}$ for the inner cone and $44.5^{\circ}$ and $50^{\circ}$ for the outer one. These close angle cones are separated in the azimuthal direction, but they are 
not distinguished in the 2D calculations. The calculated axial intensity line-outs also shown in Fig. 3c are taken half distance between the capsule and the hohlraum wall. A possible way of balancing measured emission levels of the inner and outer ring that we are considering is the use of a thinner hohlraum wall on the central part than in the area close to the LEH. Moreover, the minimum wall thickness, required for high levels of detected $\mathrm{x}$-ray flux, is given by thermal radiation burn-through and wall break-up that limit hohlraum radiation confinement used to drive the capsule. Henceforth, we performed 1D LASNEX calculations of hohlraum wall slabs with variable thickness heated by ICF $\mathrm{x}$-ray. Figure 4 shows the thin wall motion heated by an ICF-shaped radiation drive for 5 and 10 um thick high- $Z$ cocktails wall backed by 150 um of plastic $(\mathrm{CH})$. According to these 1D calculations, 10 um wall fully contains the radiation drive, while for 5 um burn-through occurs at the end of the radiation drive. This shows that a thickness slightly larger than 5 um is enough to prevent burn-through of the wall heated by thermal x-rays. Complex 2D and 3D calculations as well as experiments are necessary in the near future to assess the effects of additional laser burn-though and wall break-up due to plasma instabilities in order to validate the required hohlraum wall thickness.

\section{Thin wall photometrics and accuracy assessments for future NIF experiments}

We have begun to assess the first thin wall experimental design for NIF and photometrics estimates based on the calculated emissivities (Fig. 3). The detector of choice will be an x-ray gated camera ${ }^{9}$, positioned $90 \mathrm{~cm}$ away from the hohlraum. A magnification of 0.9 will be used to image the entire hohlraum wall at four different times during the laser pulse. Table 1 shows the calculated x-ray photometrics and accuracies for both inner and 
outer rings during the peak and rise of the laser pulse (Fig. 3a) using calculated x-ray emissivities (Fig. 3b) transmitted though 5 and 10 um cocktail wall. In order to estimate the number of photons detected and the signal-to-noise we assumed an average detected x-ray photon energy of $10 \mathrm{keV}$. Furthermore, we will optimize the pinhole size and camera exposure for a good photon statistics. The beam centroiding accuracy is given by the resolution element divided by the signal-to-noise ratio to which we added the effect of camera magnification error of 25 um which is $1 \%$ of the inter-cone distance.

Finally, the beam pointing accuracy measured by this technique for the inner and outer cones will give errors in $\mathrm{P} 2$ and $\mathrm{P} 4$ asymmetries estimated in $^{2}$ for the ICF design using 1.8 MJ of laser energy. In Table 1 we rescaled the pointing distance that gives a certain asymmetry to the smaller target dimensions of the $1 \mathrm{MJ}$ energy ICF design and compared it to the measured centroiding accuracy.

During the peak of the laser drive the emitted x-ray flux is high enough to allow us to use $\sim 70$ um pinholes and 0.1-0.2 ns camera exposure and still obtain $\sim 1000$ photons per resolution element. As shown in Fig. 3c, the inner ring emission flux is about 10 times smaller that for the outer ring, but twice thinner wall at the inner ring balances the detected signal levels as shown in Table 1. The signal-to-noise at the peak of the laser pulse is in the order of 10-20. This gives a beam centroiding accuracy of about 15 um without and 30 um with a magnification error of $1 \%$, which will generate an error in P2 inferrence of $0.4 \%$ and of $0.2 \%$ in P4.

The x-ray emission on the pulse rise (Fig. $3 b, 10 \mathrm{~ns}$ ), however, is about 5 orders of magnitude lower that for the peak. Therefore the pinhole size has to be increased to 0.5 $\mathrm{mm}$ for a camera exposure of $1 \mathrm{~ns}$ to obtain measurable signal levels. This decreases the 
centroiding accuracy to 230 um for the inner and 60 um for the outer cones. In consequence the $\mathrm{P}$ mode accuracies decrease to $2-3.5 \%$ for the inner and to about $1 \%$ for the outer cones.

With already low signal levels on the pulse rise, the thin wall imaging cannot be used during the foot of the ICF pulse (Fig. 3a) due to the low spot x-ray emission, according to calculations. Table 1 also shows that while the total x-ray conversion efficiency in the outer cone is of about $80 \%$ at all times, the hard x-ray conversion efficiency increases from $4 \mathrm{e}-5 \%$ at $10 \mathrm{~ns}$ to $0.3 \%$ at $13 \mathrm{~ns}$ on the peak of the laser pulse.

\section{Summary}

We performed the first photometrics calculations of thin wall imaging for future NIF experiments. Measured multi-cone spot emission ratio is confirmed by calculations and analytical estimates. The 2D calculations show that thin wall imaging can be used for laser spot motion during the rise of the ICF pulse (Fig 3a, $\mathrm{t}>10 \mathrm{~ns}$ ), but cannot be used on

the pulse foot. Preliminary experiments at the Omega laser facility will be used to validate the x-ray emission dependence on the laser intensity for ICF intensities. Burnthrough and wall break-up will be tested experimentally for wall thickness validation. We will also assess the possibility to identify beam filamentation and cross beam energy transfer from thin wall imaging of x-ray spot intensity variations.

\section{References}

[1] L.J. Suter et al., Rev. Sci. Instrum 68(1), 838, 1997

[2] J.D. Lindl, Phys. Plasmas 2, 3933 (1995).

[3] G.H. Miller, E.I. Moses and C.R. Wuest, Nucl. Fusion 44, 228 (2004).

[4] W.L. Kruer, The Physics of Laser Plasma Interactions, Addison-Wesley Publishing (1988). 
[5] [1] T. R. Boehly et al., Rev. Sci. Instrum. 66, 508 (1995).

[6] E.L. Dewald, L.J. Suter, O.L. Landen et al., Phys. Rev. Lett. 95, 215004, (2005).

[7] G.B. Zimmerman and W.L. Kruer, Comm. Plasma Phys. Contr. Fusion 2, 51 (1975).

[8] ICF design with LEH shields

[9] K.S. Budil et al, Rev. Sci. Instrum. 67, 485 (1996)

This work was performed under the auspices of the U. S. Department of Energy by University of California, Lawrence Livermore National Laboratory under contract W-7405-Eng-48. 
Table 1 Photometrics and accuracies for future NIF ICF hohlraums using 2D LASNEX emissivities

\begin{tabular}{|c|c|c|c|c|c|}
\hline \multirow[b]{2}{*}{ Parameter } & \multirow{2}{*}{$\begin{array}{c}\text { ICF intensity } \\
\text { Units }\end{array}$} & \multicolumn{2}{|c|}{ peak 1e15@10 ns } & \multicolumn{2}{|c|}{ rise $\sim 1 e 14 @ 13 n s$} \\
\hline & & $\begin{array}{c}\text { Inner cone, } \\
23.5\end{array}$ & $\begin{array}{c}\text { Outer cone } \\
50\end{array}$ & $\begin{array}{c}\text { Inner } \\
\text { cone }_{t} 23.5\end{array}$ & $\begin{array}{c}\text { Outer cone, } \\
50\end{array}$ \\
\hline Laser spot intensity & $\mathbf{W} / \mathrm{cm}^{\wedge} \mathbf{2}$ & $5.7 E+14$ & $9.7 \mathrm{E}+14$ & $3.0 \mathrm{E}+14$ & $2.0 \mathrm{E}+14$ \\
\hline Wall thickness 1 \& 2 & um & $5 \& 10$ & $5 \& 10$ & $5 \& 10$ & $5 \& 10$ \\
\hline Average Photon energ & keV & 10.0 & 10.0 & 10.0 & 10.0 \\
\hline Spot size, $x$-rays & $\mathrm{mm}>10(50) \%$ & $1.4(0.5)$ & $1(0.75)$ & & \\
\hline Flux through wall 1 & $W / \mathrm{cm}^{\wedge} \mathbf{2 . s r}$ & $2.2 \mathrm{E}+10$ & $2.0 \mathrm{E}+11$ & $3.0 E+05$ & $4.4 \mathrm{E}+06$ \\
\hline Flux through wall 2 & $\mathrm{~W} / \mathrm{cm}^{\wedge} \mathbf{2 . s r}$ & $6.5 \mathrm{E}+09$ & $7.0 \mathrm{E}+10$ & & \\
\hline $\mathrm{x}$-ray $\mathrm{CE}, 5 \mathrm{um} \mathrm{CT}$ & $\%$ & 0.06 & 0.29 & 3.E-06 & 4.E-05 \\
\hline X-ray CE, total & $\%$ & & 86.3 & & 77.9 \\
\hline Exposure Time & seconds & 2.E-10 & 2.E-10 & 1.E-09 & 1.E-09 \\
\hline Pinhole diameter & $\mu \mathrm{m}$ & 70 & 70 & 500 & 500 \\
\hline Resolution @ Ball & $\mu \mathrm{m}$ & 152 & 152 & 1088 & 1088 \\
\hline \# Phot. / res el. 5 um & $N / n$ & 400 & 3600 & 70.0 & 1000 \\
\hline \# Phot./res el. 10u m & $\mathbf{N} / \mathbf{n}$ & 120 & 1300 & & \\
\hline SNR per res/element & SNR & 11 & 26 & 5 & 22 \\
\hline SNR per res/element & SNR & 6 & 20 & & \\
\hline Centroiding Accuracy & $\mu \mathrm{m}$ & 14 & 6 & 225 & 50 \\
\hline Centroiding Accuracy & $\mu \mathrm{m}$ & 25 & 8 & & \\
\hline Centr. acc. $\mathrm{dM}=1 \%$ & $\mu \mathrm{m}$ & 30 & 26 & 230 & 56 \\
\hline Centr. acc. $\mathrm{dM}=1 \%$ & $\mu \mathrm{m}$ & 35 & 26 & & \\
\hline$P 2$ acc. $w$ dM=1\% & $\%$ & 0.4 & 0.4 & 3.3 & 0.8 \\
\hline$P 4$ acc. $w$ dM=1\% & $\%$ & -0.2 & -0.2 & -1.8 & -0.4 \\
\hline
\end{tabular}




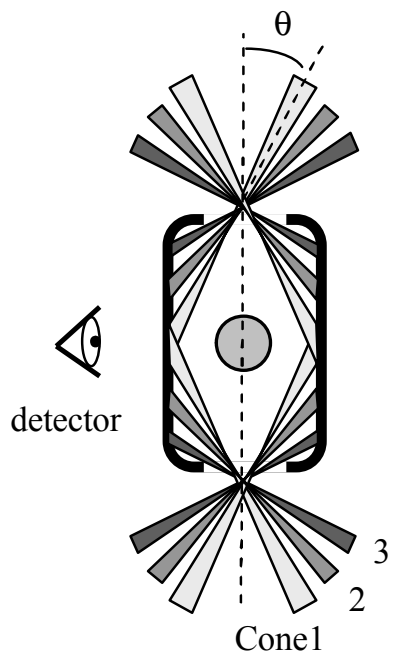

a

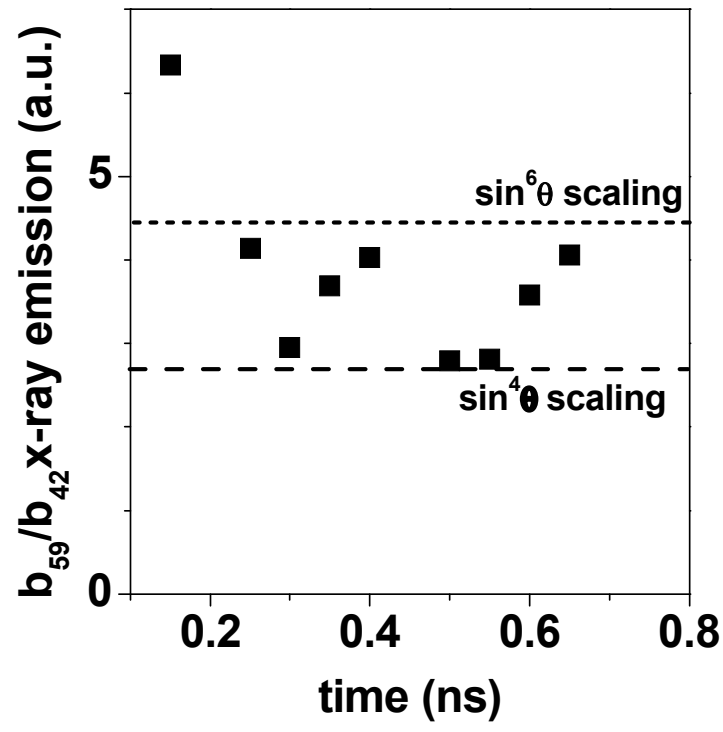

b

Figure 1 (a) Omega multi-cone laser illumination and (b) comparison of measured and analytic x-ray flux ratio estimate vs cone angle for 59 and 42 degree beams. 

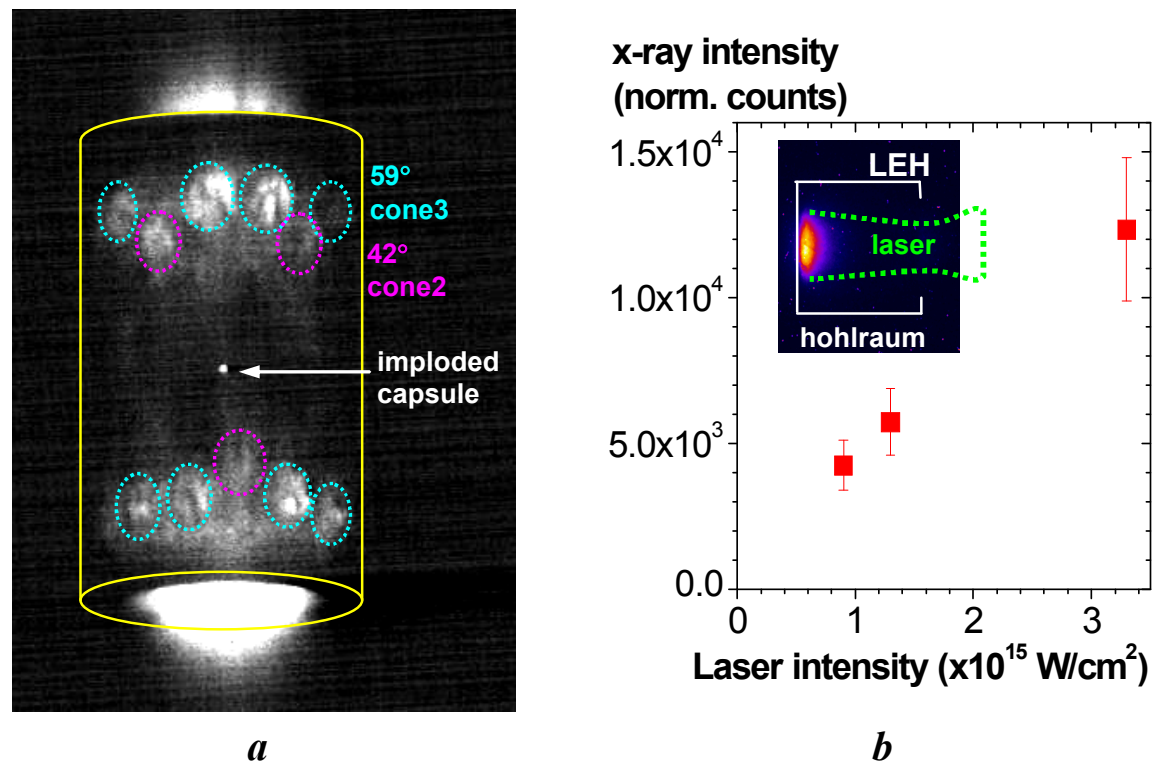

Figure 2 (a) Time integrated x-ray emission measured in Omega implosion experiments and (b) gated thin wall images and x-ray intensity vs laser intensity recorded during first NIF hohlraum experiments. 

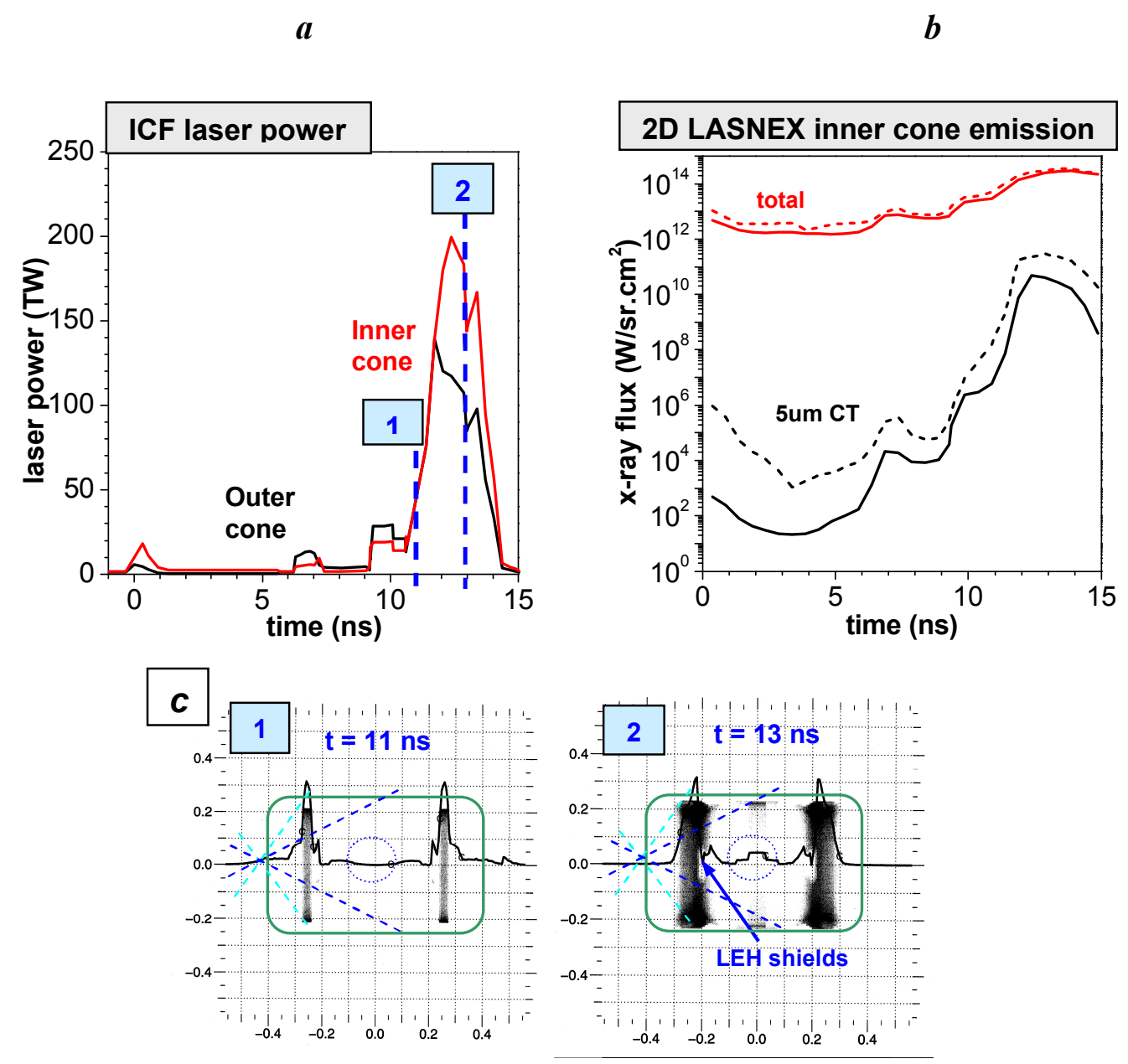

Figure 3 2D LASNEX calculations of 1 MJ ICF design with LEH shields: (a) total laser power in the inner and the outer cones and (b) calculated inner and outer cone x-ray emission vs time: unfiltered, and transmitted through 5 um cocktail wall; (c) Postprocessed x-ray emission in the hohlraum for two times during the ICF pulse. 


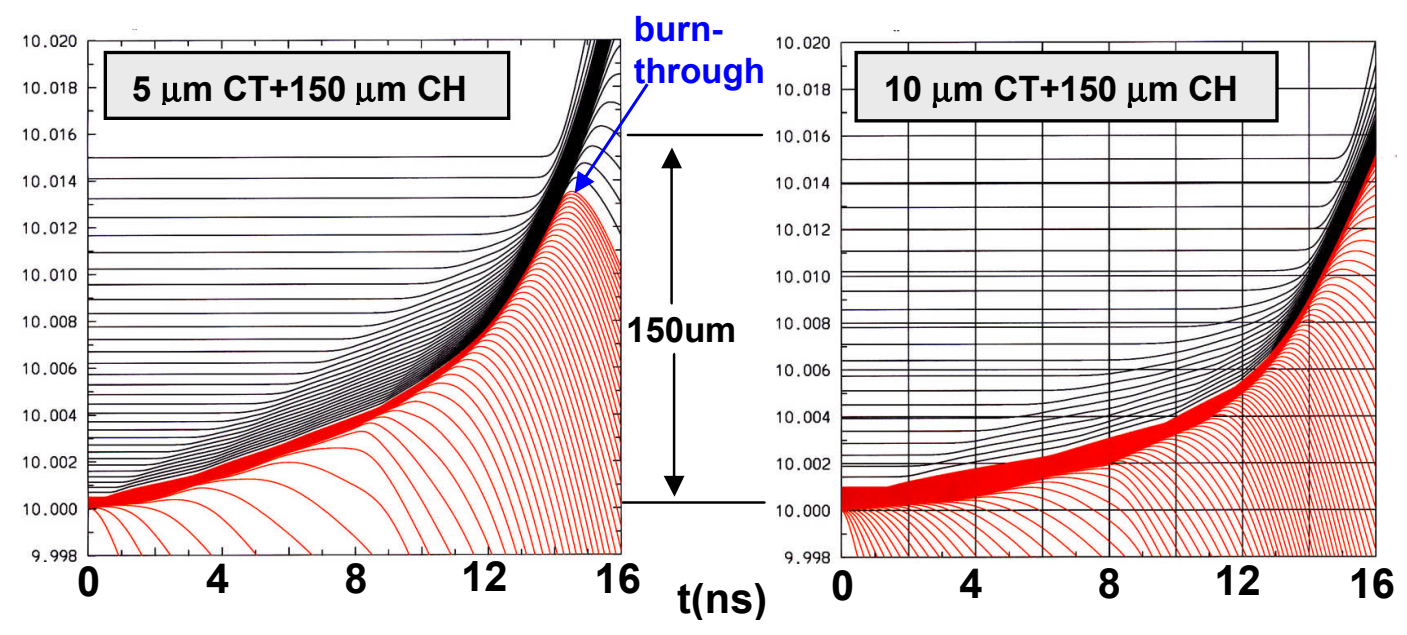

Figure 4 Dynamics of thin wall hohlraum wall heated by ICF-like x-ray drive calculated with 1D LASNEX; while burn-through occurs for 5 um cocktail wall at the end of the drive (14 ns), the radiation is fully confined for 10 um wall. 